\title{
Long-term Follow-up of Dorsal Wedge Osteotomy for Pediatric Freiberg Disease
}

Foot \& Ankle International 2016, Vol. 37(I) 90-95 (C) The Author(s) 2015 Reprints and permissions: sagepub.com/journalsPermissions.nav DOI: I0.1 I77/I07|I007/5598602

fai.sagepub.com

\author{
Bruno S. Pereira, MD', Tiago Frada, MD ${ }^{1,2}$, Daniel Freitas, MD', \\ Pedro Varanda, MD', Manuel Vieira-Silva, MD', Xavier Martin Oliva, MD, PhD ${ }^{4,5}$, \\ and Rui M. Duarte, MD ${ }^{1,2,3}$
}

\begin{abstract}
Background: Treatment for Freiberg disease has been largely conservative despite availability of various operative options for severe or refractory cases. The aim of this study was to evaluate the long-term results of pediatric patients with symptomatic Freiberg disease treated with intra-articular dorsal wedge osteotomy.

Methods: Pediatric patients treated for Freiberg disease with surgery between January 1982 and 1999 were identified and selected for long-term clinical evaluation. Patients were evaluated regarding operative satisfaction and clinical outcome, performed according to the American Orthopaedic Foot \& Ankle Society (AOFAS) lesser toe metatarsophalangealinterphalangeal scale and range of motion (ROM) of metatarsophalangeal (MTP) joint. Patients had radiographic assessment of degenerative joint status with anteroposterior and oblique foot x-ray. Twenty patients ( 18 female, 2 male; mean age 15.2 years; range 12-17 years) were identified. The mean follow-up period was 23.4 (range 15-32) years.

Results: The clinical outcomes of our patients were classified as excellent in $16(80 \%)$ and good in $4(20 \%)$. The AOFAS mean score was 96.8 (range 9I-100) points at the last clinical appointment. A negative correlation between AOFAS score and time of follow-up (r's $=-0.6 \mathrm{I}, P<.00 \mathrm{I}$ ) was found. Also, a strong negative correlation was found between Smillie classification and AOFAS final score $(r$ 's $=-0.88, P<.001)$.

Conclusion: The patients were very satisfied with pain and quality of life at a mean follow-up time of 23.4 years. To our knowledge, this is the first long-term follow-up report supporting the procedure described by Gauthier and Elbaz as a good option for operative treatment of Freiberg disease.

Level of Evidence: Level IV, retrospective case series.
\end{abstract}

Keywords: forefoot disorders, Freiberg disease, Gauthier and Elbaz, metatarsal heads, dorsal wedge osteotomy

\section{Introduction}

Alfred Freiberg, in 1914, was the first to identify a medical condition that affected the second metatarsal head. ${ }^{8} \mathrm{He}$ reported a series of 6 patients complaining of mechanical pain over the second metatarsal region. ${ }^{8}$ Further radiographic study revealed a sclerotic and flattened metatarsal head, described as an osteochondrosis of the metatarsal head, currently known as Freiberg disease. ${ }^{2,3}$ Although it is a relatively rare condition, it has been recognized as one of the most common osteochondrosis of the body. ${ }^{20}$ It occurs typically during adolescence, affecting females most often $(5: 1)^{5,13}$ Although osteochondrosis can affect all metatarsal heads, $68 \%$ of cases relate to the second metatarsal head, whereas $27 \%$ occur in the third and $3 \%$ in the fourth. ${ }^{2,6}$ Bilateral involvement can be expected in $6.6 \%$ of patients. ${ }^{2,9,25}$

One century after the first description of the disease, the etiology remains unclear. Multiple mechanisms have been proposed, but the multifactorial etiology resulting from a combination of trauma, impaired vascularity, genetic disorders, and/or altered biomechanics is the most accepted. ${ }^{2,6,23,25}$

Radiographic examination is suitable to identify metatarsal head changes along the course of the disease. Smillie in $1967^{22}$ categorized the macroscopic appearance of the

'Orthopedic Surgery Department, Hospital de Braga, Braga, Portugal ${ }^{2}$ Life and Health Sciences Research Institute (ICVS), School of Health Sciences, University of Minho, Braga, Portugal

${ }^{3}$ ICVS/3Bs - PT Government Associate Laboratory, Braga/Guimarães, Portugal

${ }^{4}$ Orthopedic Surgery Department, Clínica Ntra. Sra. Del Remei, Carrer de l'Escorial, Barcelona, Espanha

${ }^{5}$ Facultad de Medicina, University of Barcelona, Casanova, Barcelona, Espanha

Corresponding Author:

Bruno S. Pereira, MD, Hospital de Braga - Orthopedic Surgery

Department, Sete Fontes - São Victor, 47 I0-243 Braga, Portugal.

Email: pereira.bac@gmail.com 
metatarsal head into 5 stages: stage I, early fracture of the subchondral epiphysis; stage II, early collapse of the dorsal central portion of the metatarsal head with flattening of the joint surface; stage III, further flattening of the metatarsal head with continued collapse of the central portion of the joint surface with a medial and lateral projection, while the plantar articular cartilage remains intact; stage IV, loose body formation originating from fractures of the lateral projection and separation of the central articular fragment; stage $\mathrm{V}$, end-stage degenerative arthrosis with marked flattening of the metatarsal head and joint space narrowing. ${ }^{22}$ This classification is the most popular and provides a guide to defining treatment options. ${ }^{2,3}$

The primary treatment for Freiberg disease consists of conservative measures, such as activity restriction and the use of orthotics or casts. ${ }^{24}$ When conservative treatment fails, operative treatment should be considered. Many operative procedures have been described, including resection arthroplasty, joint debridement, osteotomy and osteochondral transplant. Nonetheless, no consensus regarding which is the best operative approach has been reached to date. ${ }^{2}$

Dorsal wedge or dorsiflexion osteotomy, described in 1979 by Gauthier and Elbaz, has shown good results regardless of the stage of the disease. ${ }^{9,15}$ The operative procedure includes a dorsal closing wedge osteotomy through the involved metatarsal head, excising the damaged joint cartilage and allowing the intact plantar cartilage to articulate with the proximal phalanx. Because this operative technique has been described, many authors have reported on the success of dorsal wedge osteotomies. ${ }^{3,7,8,14,16,20,23}$ The current study aimed to evaluate the long-term results of pediatric patients with symptomatic Freiberg disease treated with intra-articular dorsal wedge osteotomy.

\section{Methods}

We retrospectively reviewed 23 pediatric patients with the diagnosis of Freiberg disease that were operatively treated by the technique described by Gauthier and Elbaz ${ }^{9}$ in our institution between January 1982 and January 1999. From the total of 23 patients that met the inclusion criteria, 20 entered the study, whereas 3 patients were unable to be contacted. The patients' mean age at surgery was 15.2 (range 12-17) years, with a mean follow-up of 23.4 (range 15-32) years. The right foot was most commonly affected (14 patients) and no bilateral disease was verified. Radiographic assessment categorized 8 patients in stage II, 9 patients in stage III, and 3 patients in stage IV. The second metatarsal was involved in 17 patients, and the third in 3 (Table 1).

The operative technique was performed under regional or general anesthesia, and a tourniquet was used. A dorsal incision over the metatarsophalangeal joint was performed. The joint was debrided, and any loose bodies or hypertrophic synovium were removed. The lesion on the articular surface
Table I. Demographic data of patients treated for Freiberg disease and operatively submitted to Gauthier and Elbaz osteotomy between January 1982 and January 1999.

\begin{tabular}{lc}
\hline Age at surgery, y (SD) & I5.2 $(+/-1.6)$ \\
Gender (male/female) & $2: 18$ \\
Laterality (R/L) & $14: 6$ \\
Metatarsal involved & $17: 3$ \\
$\quad$ (second/third) & \\
Follow-up, y (SD) & $23.4(4.6)$ \\
Smillie classification & \\
I & 0 \\
II & 8 \\
III & 9 \\
IV & 3 \\
V & 0 \\
\hline
\end{tabular}

Abbreviations: L, left; R, right; SD, standard deviation.

was excised, followed by a dorsal wedge osteotomy. The wedge size was directly related with the lesion size (Figure 1). The metatarsal head was rotated dorsally and proximally. The length of the metatarsal was preserved as much as possible. Internal fixation was performed with a figure-of- 8 stainless steel wire loop. A posterior ankle splint was applied, which was discontinued at 6 weeks. Then the patients were instructed to use soft shoes and avoid strenuous activities for 8 weeks.

All patients failed conservative treatment defined by persistent pain after 6 months of treatment. Diagnosis was defined by clinical and radiographic assessment. Clinical outcomes were evaluated according to the American Orthopaedic Foot \& Ankle Society (AOFAS) lesser toe metatarsophalangeal-interphalangeal scale, range of motion (ROM) of metatarsophalangeal (MTP) joint, and the patient's subjective satisfaction (poor, good, excellent). Complications were also recorded.

Patients were evaluated by radiographic assessment of degenerative joint status with anteroposterior and oblique foot x-ray.

Statistical analysis was performed with IBM SPSS Statistics 22.0 (IBM Corp, Armonk, NY). Spearman correlation test was performed to test the association between the AOFAS score and follow-up time and between Smillie classification and AOFAS final score.

\section{Results}

There were no complications observed such as infection, non-union, or malunion. The AOFAS mean score was 96.8 (range 91-100) points at the last clinical appointment (Figure 2). In the same clinical encounter, patients were asked to subjectively define their level of satisfaction with the procedure. All patients were satisfied with the final outcome of the procedure, with $16(80 \%)$ reporting excellent results and $4(20 \%)$ good results. 


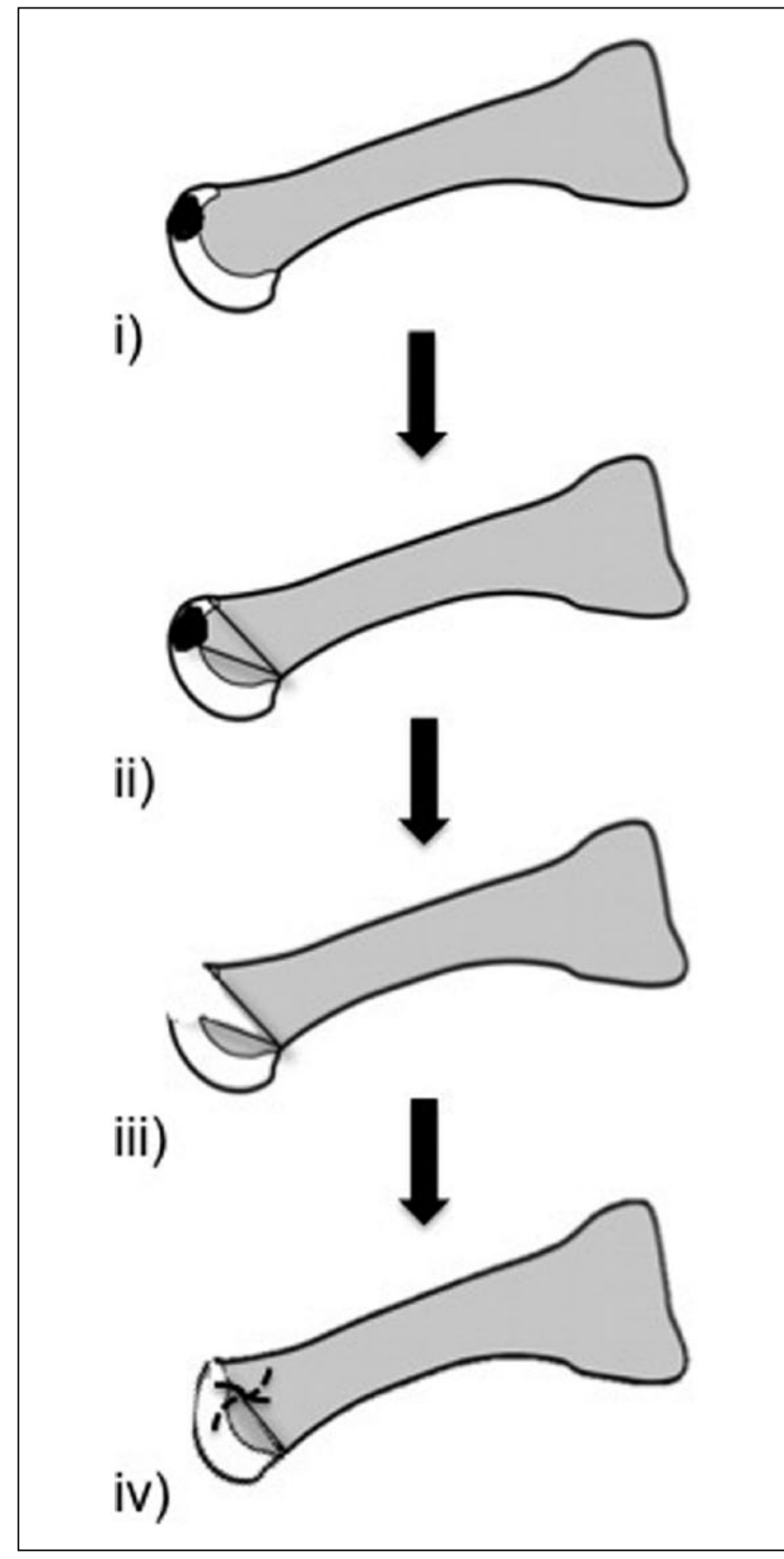

Figure I. Diagram of the operative technique: (i) identification of lesion; (ii) design of wedge osteotomy; (iii) lesion excision; and (iv) metatarsal head dorsal rotation and internal fixation.

A negative correlation was found between AOFAS score and time of follow-up (r's $=-0.61, P<.001$ ). Also, when assessing the relationship between Smillie classification and AOFAS final score, a strong negative correlation was found (r's $=-0.88, P<.001$ ).

Radiographic assessment demonstrated a good intraarticular space and metatarsal head coverage and contour. These findings are highlighted in Figures 3 to 5. Preoperative imaging (A) is compared with postoperative imaging at time of surgery (B) and at follow-up evaluation (C).

\section{Discussion}

Since Freiberg described metatarsal head osteochondrosis, classification systems and treatment procedures have not been clearly established. ${ }^{2}$ Classifications are usually based on vascular influence and radiographic degenerative changes. $^{22}$ Smillie's stages I and II are usually amenable to conservative measures for a period of at least 6 months. An operative approach should be reserved for stages III, IV, and $V^{7,14,22}$ Despite the advent of new operative techniques, such as osteochondral plug transfer, arthroplasty or arthroscopic treatment, the ideal method to treat Freiberg disease remains unanswered. $5,6,11,14,16,18,19,21$

Dorsal closing-wedge osteotomy was first described by Gauthier and Elbaz in 1979 providing good results in 52 out of 53 patients. ${ }^{9}$ After the first description of this operative technique, many authors reported clinical data supporting the success of dorsal wedge osteotomies. $1,4,10,12,17,26$ Lee et al in their study with 13 patients showed a mean AOFAS score of 92.2, and no complications. ${ }^{16}$ Chao et al with a mean follow-up of 40 months in 13 patients reported no complications and 4 excellent results and 7 good results. ${ }^{4}$ Capar et al published excellent results in 7 patients, good in 9 patients, poor in 3 patients, and no complications. ${ }^{1}$ Nonetheless, there have been no longterm results to identify transfer metatarsalgia or other complications.

The present study reviewed the long-term outcome of dorsal closing wedge osteotomy performed in a pediatric population, providing valuable information on how effective this procedure can be for the treatment of Freiberg disease.

Our results demonstrated that patients were very satisfied (16 excellent, 4 good results) concerning postprocedure pain and quality of life at a mean follow-up time of 23.4 years. These good subjective results could be influenced by the initial intermediate degree of degenerative changes in our study population ( $85 \%$ in stage II and III). Nonetheless, the long-term follow-up provides evidence that results can be sustained for the long run.

The final AOFAS mean score was also very high $96.8 / 100$, ranging from 91 to 100 . Achieving such results for such a long period is a strong support of the operative procedure. There was a strong negative correlation between AOFAS score and time of follow-up, meaning we could expect some worsening over time. Also, the AOFAS final score correlated negatively with the initial Smillie stage. Nonetheless, final scores ranging from 91 to 100 points at 23.4 years clearly show that these findings are probably clinically negligible. In the present study, we had no complications such as non-union, infection, or transfer metatarsalgia.

The present study had some limitations, including the retrospective design and the small number of patients that entered the study. Still, the power of our very long follow-up 


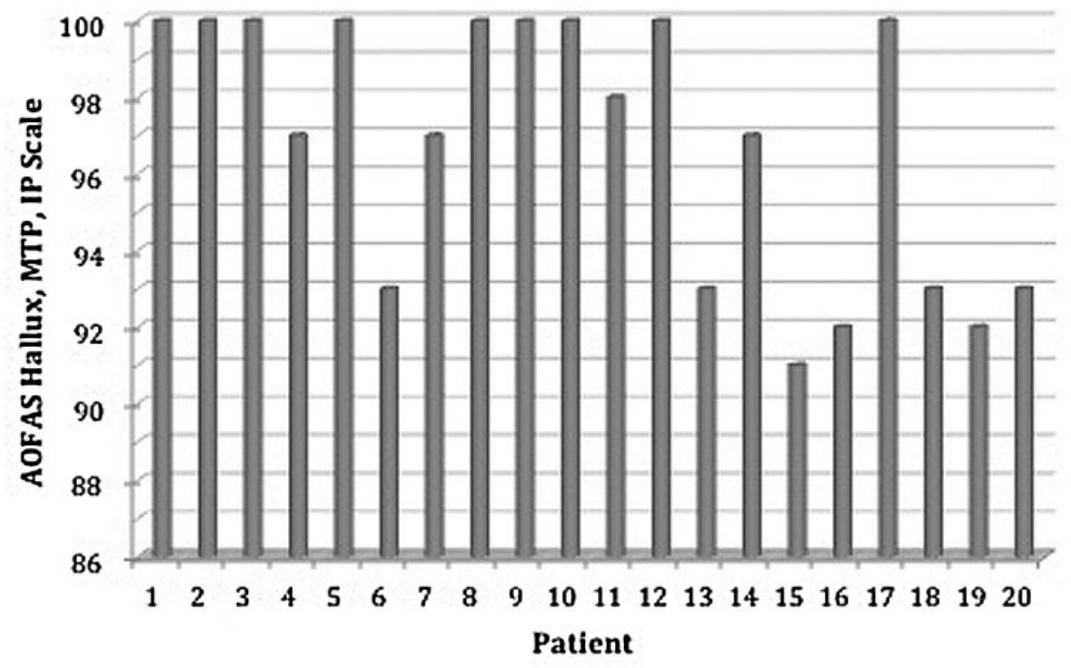

Figure 2. Individual AOFAS score at last follow up.
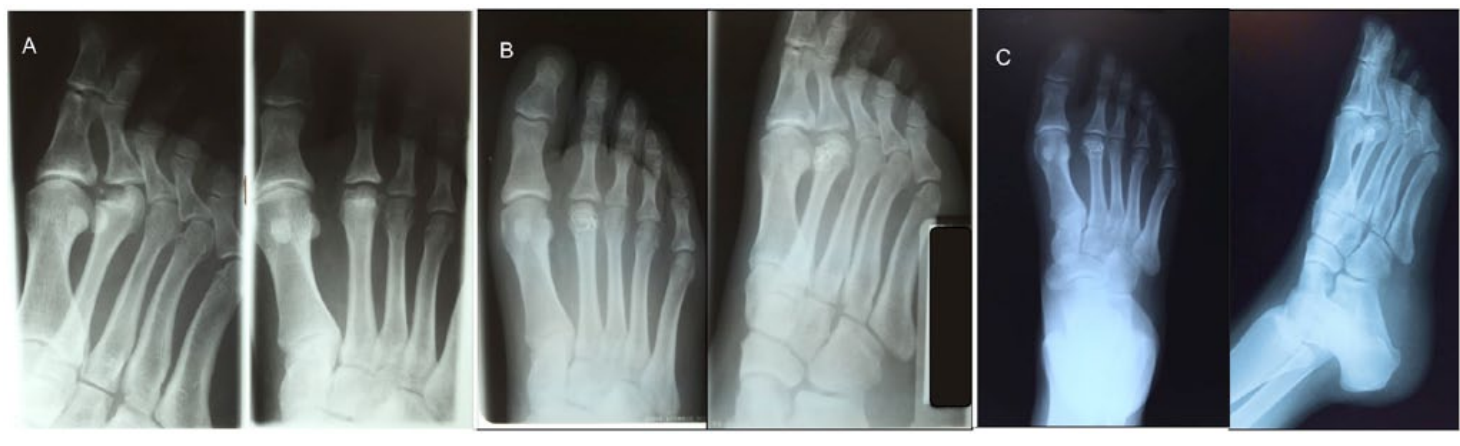

Figure 3. X-ray film of right foot in 16-year-old girl. Preoperative imaging $(A)$ is compared with postoperative imaging at time of surgery $(B)$ and at follow-up evaluation-26 years $(C)$.

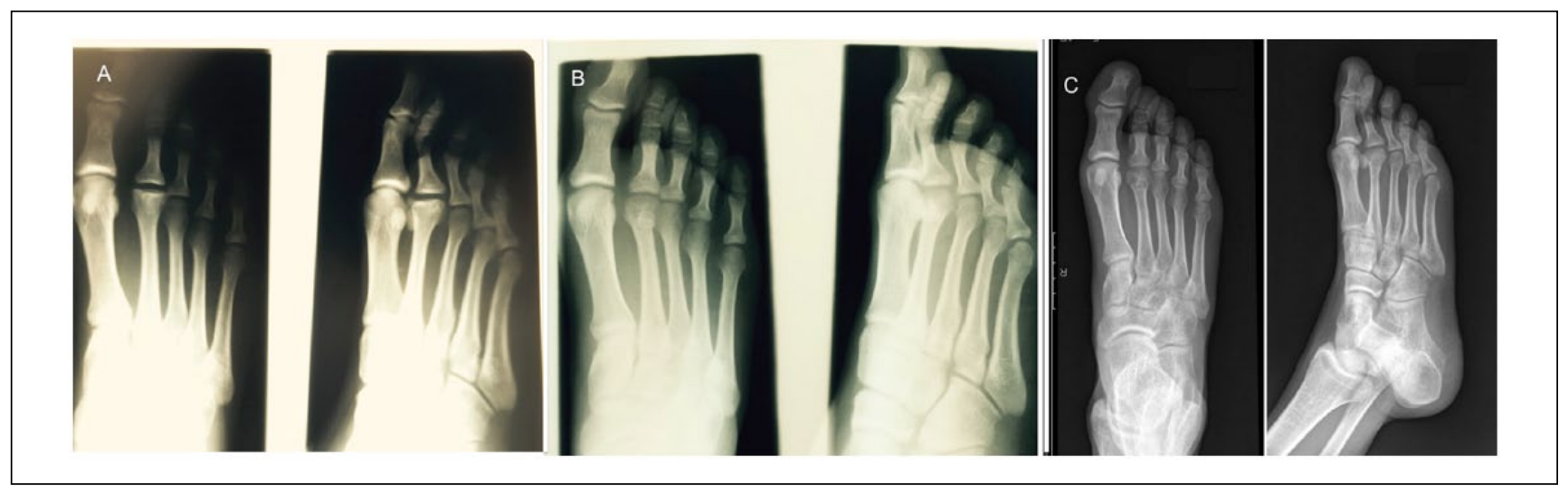

Figure 4. X-ray film of right foot in 17-year-old boy. Preoperative imaging $(A)$ is compared with postoperative imaging at time of surgery $(B)$ and at follow-up evaluation-23 years $(C)$. 

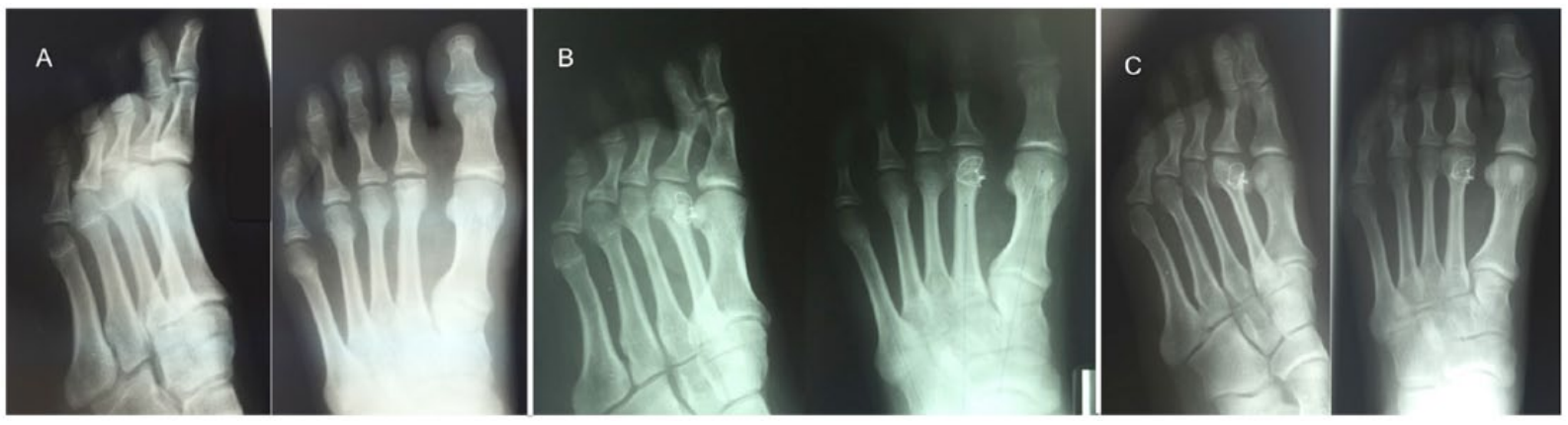

Figure 5. X-ray film of right foot in I4-year-old girl. Preoperative imaging $(A)$ is compared with postoperative imaging at time of surgery $(B)$ and at follow-up evaluation-25 years $(C)$.

time and the unique characteristics of the population are its strength. To our knowledge, this is the first study on operative treatment of Freiberg disease in a pediatric population and the first with such a long follow-up concerning the dorsal wedge osteotomy procedure described by Gauthier and Elbaz.

\section{Declaration of Conflicting Interests}

The author(s) declared no potential conflicts of interest with respect to the research, authorship, and/or publication of this article.

\section{Funding}

The author(s) received no financial support for the research, authorship, and/or publication of this article.

\section{References}

1. Capar B, Kutluay E, Müjde S. Dorsal closing-wedge osteotomy in the treatment of Freiberg's disease [in Turkish]. Acta Orthop Traumatol Turc. 2007;41:136-139.

2. Carmont MR, Rees RJ, Blundell CM. Current concepts review: Freiberg's disease. Foot Ankle Int. 2009;30(2): 167-176.

3. Cerrato RA. Freiberg's disease. Foot Ankle Clin. 2011;16: 647-658.

4. Chao KH, Lee $\mathrm{CH}$, Lin LC. Surgery for symptomatic Freiberg's disease: extraarticular dorsal closing-wedge osteotomy in 13 patients followed for 2-4 years. Acta Orthop Scand. 1999;70:483-486.

5. DeVries JG, Amiot RA, Cummings P, Sockrider N. Freiberg's infraction of the second metatarsal treated with autologous osteochondral transplantation and external fixation. J Foot Ankle Surg. 2008;47:565-570.

6. Edmondson MC, Sherry KR, Afolyan J, Armitage AR, Skyrme AD. Case series of 17 modified Weil's osteotomies for Freiberg's and Köhler's II AVN, with AOFAS scoring pre- and post-operatively. Foot Ankle Surg. 2011;17: 19-24.
7. Espinosa N, Maceira E, Myerson MS. Current concept review: metatarsalgia. Foot Ankle Int. 2008;29(8): 871-879.

8. Freiberg AH. Infraction of second metatarsal bone, atypical injury. Surg Gynaecol Obstet. 1914;19:191.

9. Gauthier G, Elbaz R. Freiberg's infraction: a subchondral bone fatigue fracture. A new surgical treatment. Clin Orthop Relat Res. 1979;142:93-95.

10. Gong HS, Baek GH, Jung JM, Kim JH, Chung MS. Technique tip: fixation of dorsal wedge osteotomy for Freiberg's disease using bioabsorbable pins. Foot Ankle Int. 2003;24(11): 876-877.

11. Hayashi K, Ochi M, Uchio Y, Takao M, Kawasaki K, Yamagami N. A new surgical technique for treating bilateral Freiberg disease. Arthroscopy. 2002; 18:660-664.

12. Ikoma K, Maki M, Kido M, et al. Extra-articular dorsal closing-wedge osteotomy to treat late-stage Freiberg disease using polyblend sutures: technical tips and clinical results. Int Orthop. 2014;38:1401-1405.

13. Katcherian DA. Treatment of Freiberg's disease. Orthop Clin North Am. 1994;25:69-81.

14. Kilic A, Cepni KS, Aybar A, Polat H, May C, Parmaksizoglu AS. A comparative study between two different surgical techniques in the treatment of late-stage Freiberg's disease. Foot Ankle Surg. 2013;19:234-238.

15. Kinnard P, Lirette R. Freiberg's disease and dorsiflexion osteotomy. J Bone Joint Surg Br. 1991;73:864-865.

16. Lee HJ, Kim JW, Min WK. Operative treatment of Freiberg disease using extra-articular dorsal closing-wedge osteotomy: technical tip and clinical outcomes in 13 patients. Foot Ankle Int. 2013;34(1):111-116.

17. Lee SK, Chung MS, Baek GH, Oh JH, Lee YH, Gong HS. Treatment of Freiberg disease with intra-articular dorsal wedge osteotomy and absorbable pin fixation. Foot Ankle Int. 2007;28(1):43-48.

18. Maresca G, Adriani E, Falez F, Mariani PP. Arthroscopic treatment of bilateral Freiberg's infraction. Arthroscopy. 1996;12:103-108.

19. Miyamoto W, Takao M, Uchio Y, Kono T, Ochi M. Late-stage Freiberg disease treated by osteochon- 
dral plug transplantation: a case series. Foot Ankle Int. 2008;29(9):950-955.

20. Omer GE. Primary articular osteochondroses. Clin Orthop. 1981;158:33.

21. Ozkan Y, Oztürk A, Ozdemir R, Aykut S, Yalçin N. Interpositional arthroplasty with extensor digitorum brevis tendon in Freiberg's disease: a new surgical technique. Foot Ankle Int. 2008;29(5):488-492.

22. Smillie IS. Treatment of Freiberg's infraction. Proc $R$ Soc Med. 1967;60:29-31.
23. Stanley D, Betts RP, Rowley DI, Smith TW. Assessment of etiologic factors in the development of Freiberg's disease. $J$ Foot Surg. 1990;29:444-447.

24. Stanley D, Smith TWD, Rowley DI. Conservative and surgical management of Freiberg's disease. The Foot. 1991;1:97-100.

25. Talusan PG, Diaz-Collado PJ, Reach JS Jr. Freiberg's infraction: diagnosis and treatment. Foot Ankle Spec. 2014;7:52-56.

26. Xie X, Shi Z, Gu W. Late-stage Freiberg's disease treated with dorsal wedge osteotomy and joint distraction arthroplasty: technique tip. Foot Ankle Int. 2012;33(11):1015-1017. 